Typ-2-Diabetes

\title{
Aktuelle EASD-Daten: Patienten profitieren besonders von frühem Liraglutid-Einsatz
}

Bei der Therapie des Typ-2-Diabetes stehen heute individuelle Therapieziele unter Berücksichtigung von Patientenwünschen, Diabetesdauer, Komorbiditäten sowie Hypoglykämierisiko und Gewichtsentwicklung u.a. im Vordergrund. „Starre Therapieschemata wurden in den letzten Jahren immer mehr von individuellen Behandlungskonzepten abgelöst“, betonte Dr. Marcel Kaiser, Frankfurt, im Rahmen einer Pressekonferenz post-EASD und ergänzte: „Der frühe Einsatz risikoarmer Therapiestrategien wie Inkretin-basierte Behandlungsoptionen und moderne Basalinsuline mit gegenüber NPH-Insulin vermindertem Hypoglykämierisiko ermöglichen dabei eine verbesserte Diabetesbehandlung.“

Frühe Reduktion von $\mathrm{HbA}_{1 \mathrm{c}}$ und Gewicht mit GLP-1-basierten Therapieoptionen Aufgrund ihrer möglichen positiven Eigenschaften (effektive Blutzuckerkontrolle, ein in Abhängigkeit des Kombinationspartners vergleichsweise

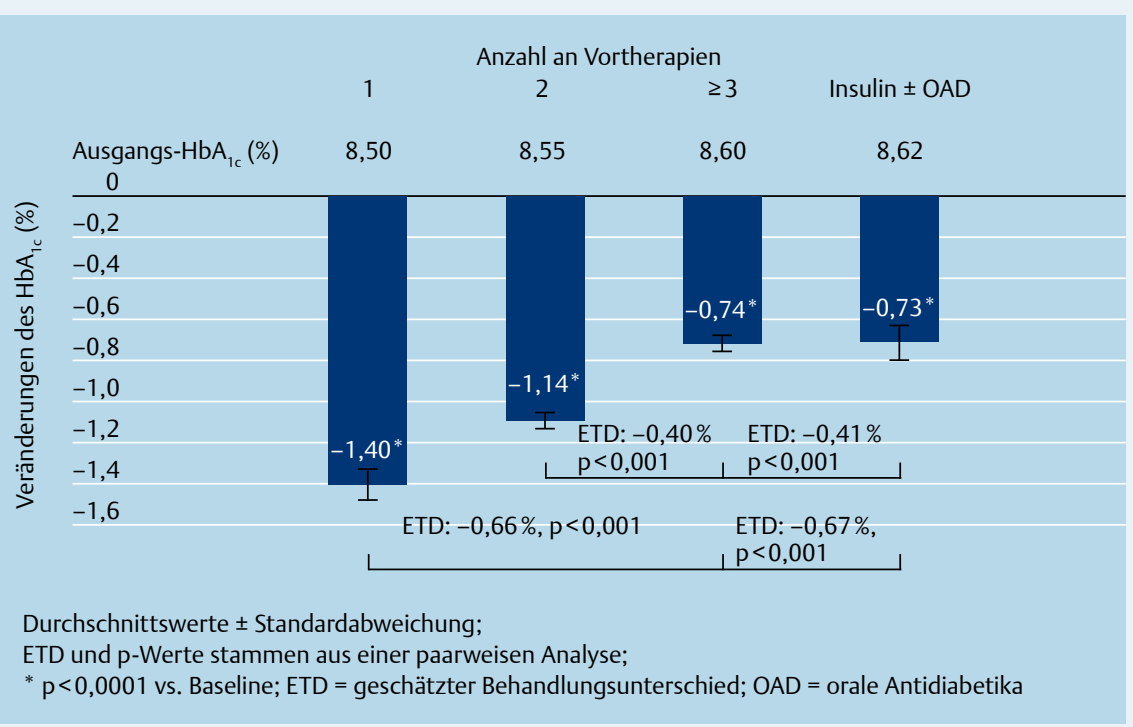

Abb. 1 Patienten profitieren von einer signifikant größeren durchschnittlichen $\mathrm{HbA}_{1 \mathrm{c}}$-Senkung unter Liraglutid, wenn sie eine weniger intensive Vortherapie erhalten haben.

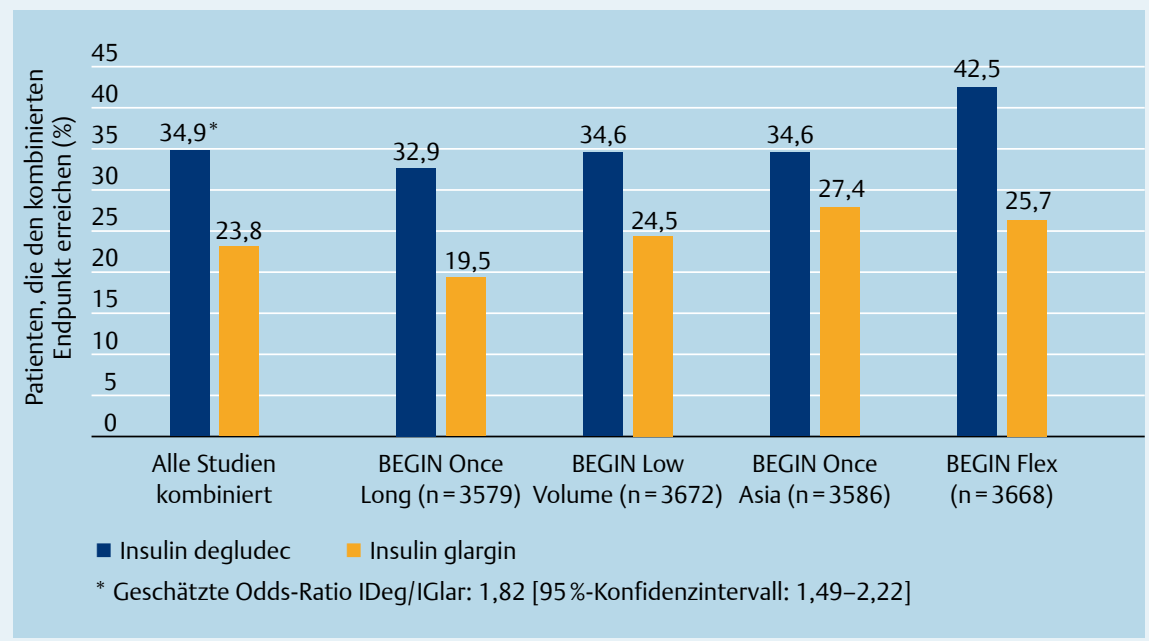

Abb. 2 Signifikant mehr Patienten unter Insulin degludec als unter Insulin glargin erreichten den NBZ-Zielwert ohne Hypoglykämien - so das Ergebnis einer gepoolten Analyse von 4 Treat-to-TargetStudien [mod. nach 12]. geringes Hypoglykämierisiko und Gewichtsreduktion) eignen sich GLP-1-Rezeptoragonisten wie Liraglutid (Victoza ${ }^{\circledR}$ ) besonders für den Einsatz in der frühen Phase des Typ-2-Diabetes. Sie werden daher im Positionspapier der amerikanischen und europäischen Diabetesgesellschaften (ADA/EASD) sowie den aktuellen Nationalen VersorgungsLeitlinien zu den möglichen Therapieoptionen direkt nach Metformin gezählt [1, 2].

Dieses Vorgehen wird durch eine beim EASD 2013 präsentierte Analyse der nicht-interventionellen retrospektiven post-Marketing Datenauswertung EVIDENCE („Case-note-Survey“) bestätigt: Patienten, die eine kürzere Diabetesdauer und weniger Vortherapie aufwiesen, profitierten besonders von einer Behandlung mit Liraglutid. So sank der $\mathrm{HbA}_{1 \mathrm{c}}$ bei Patienten, die vor Beginn der Therapie nur ein orales Antidiabetikum (OAD) eingenommen hatten, unter Liraglutid durchschnittlich um $1,4 \%$, bei Vorbehandlung mit 2 OAD um 1,14\% das ist jeweils signifikant mehr als bei Patienten, die bereits 3 oder mehr OAD zu Beginn der Liraglutid-Therapie einnahmen oder die mit Insulin vorbehandelt waren (alle p-Werte $<0,001$; Abb. 1) [3]. Neben der Intensität der Vortherapie kann auch die Erkrankungsdauer als Prädiktor für das Ansprechen auf Liraglutid herangezogen werden: Patienten mit einer Diabetesdauer von bis zu 5 Jahren bzw. zwischen 6 und 10 Jahren profitierten mit einer signifikant stärkeren $\mathrm{HbA}_{1 \mathrm{c}}$-Senkung unter Liraglutid als Patienten, deren Erkrankung schon länger als 10 Jahre anhielt [3]. „Doch auch jene Patienten, die bereits lange an Diabetes erkrankt (>10 Jahre) bzw. die intensiv vorbehandelt sind, zeigen klinisch relevante Effekte unter Liraglutid,“ resümierte Prof. Dr. Werner Kern, Ulm.

\section{Patienten bevorzugten Liraglutid}

Diese Daten stehen im Einklang mit den Ergebnissen klinischer Studien, die Liraglutid eine schnelle und langanhaltende $\mathrm{HbA}_{1 \mathrm{c}}$-Senkung sowie eine Gewichtsreduktion bestätigen [4]. Patienten, die von Sitagliptin auf Liraglutid umgestellt 
wurden, profitierten davon mit einer weiteren Abnahme des $\mathrm{HbA}_{1 \mathrm{c}}$ und des Körpergewichtes [5]. Die verbesserte glykämische Kontrolle unter Liraglutid im Vergleich zu Sitagliptin bestätigte auch eine weitere auf dem EASD präsentierte Auswertung der EVIDENCE-Studie: Nach der Umstellung vom DPP-4-Inhibitor auf den GLP-1-Rezeptoragonisten sanken $\mathrm{HbA}_{1 \mathrm{c}}$, Nüchternblutzucker (NBZ) und Körpergewicht signifikant [6]. Darüber hinaus waren die Patienten nach der Umstellung von Sitagliptin auf Liraglutid insgesamt zufriedener mit der Therapie als zuvor [5].

\section{Intensivierung mit Insulin detemir: \\ Effektive $\mathrm{HbA}_{1 \mathrm{c}}$-Kontrolle}

Bei einer Progression des Typ-2-Diabetes unter Metformin und Liraglutid kann die Behandlung durch die Zugabe des Basalinsulins Insulin detemir (Levemir ${ }^{\circledR}$ ) intensiviert werden. Aktuelle Daten von Rosenstock et al. zeigten, dass die Addition von Insulin detemir zu einem deutlichen Rückgang von $\mathrm{HbA}_{1 \mathrm{c}}$ und $\mathrm{NBZ}$ führte [7]. Kern betonte: „Trotz der Zugabe von Insulin detemir bleibt in der Regel die unter Liraglutid erzielte Gewichtsabnahme erhalten. Unter Insulin detemir traten außerdem keine schweren Hypoglykämien auf und die Raten an leichten Hypoglykämien waren mit 0,034-0,228 Ereignissen pro Patientenjahr niedrig“.
Insulin degludec vs. Insulin glargin: Kombinierter Endpunkt häufiger erreicht

Eine mögliche Alternative bei der Intensivierung der Therapie von Menschen mit Typ-2-Diabetes mittels basalunterstützter oraler Therapie (BOT) könnte Dr. Jörg Lüdemann, Falkensee, zufolge Insulin degludec (Tresiba ${ }^{\circledR}$ ) sein: Insulin degludec ist ein neues Basalinsulin mit einem stabilen Zeit-Wirkungsprofil, einer niedrigen intraindividuellen Variabilität und einem langen blutzuckersenkenden Effekt, der über 42 Stunden anhält [8-10]. In einer gepoolten retrospektiven Analyse aus 4 randomisierten offenen klinischen Treat-to-Target-Studien über 26 und 52 Wochen bei Patienten mit Typ-2-Diabetes konnten Zinman et al. zeigen, dass es unter einer BOT mit Insulin degludec um $82 \%$ wahrscheinlicher ist, einen NBZ $<90 \mathrm{mg} / \mathrm{dl}(5 \mathrm{mmol} / \mathrm{l})$ ohne bestätigte nächtliche Hypoglykämien zu erreichen als mit Insulin glargin (OR=1,82; 95\%-KI: 1,49-2,22; p<0,05; Abb. 2) [11].

Eine große Metaanalyse aus 7 klinischen Studien, die insulinbehandelte Menschen mit Typ-1- und Typ-2-Diabetes einschloss, zeigte außerdem, dass bei Insulin degludec und Insulin glargin eine größere intraindividuelle und von-Tagzu-Tag-Variabilität des NBZ mit einer höheren Rate an bestätigten Hypoglykämien bei BOT-Patienten assoziiert ist.
„Eine Reduktion dieser Variabilität könnte die Hypoglykämierate der Patienten senken“, fasste Lüdemann abschließend zusammen [12].

Monika Walter, München

\section{Literatur}

1 Inzucchi SE et al. Diab Care 2012; 35: 13641379

2 Nationale VersorgungsLeitlinie „Therapie des Typ-2-Diabetes.“ 1. Auflage, August 2013, http://www.diabetes.versorgungsleitlinien. de

3 Penfornis A et al. Diabetologia 2013; 56 (Suppl. 1): S356

4 Pratley R et al. Int J Clin Pract 2011; 65: 397407

5 Pratley R et al. Diabetes Care 2012; 35: 1986-1993

6 Charpentier G et al. Diabetologia 2013; 56 (Suppl. 1): S358

7 Rosenstock J et al. J Diabetes Complications 2013, doi:pii:S1056-8727(13)0010310.1016/j.jdiacomp. 2013.04.008

8 Heise T et al. Diabetologia 2010; 53 (Suppl. 1): $S 387$

9 Heise T et al. Diabetes 2011; 60 (Suppl. 1): A263

10 Nosek L et al. Diabetes 2011; 60 (Suppl. 1): 49-LB

11 Zinman B et al. Diabetologia 2013; 56 (Suppl. 1): $\$ 422$

12 Bode B et al. EASD 2013, Poster 1052

Quelle: Media Lunch post-EASD

Individualisierte Therapiekonzepte des

Diabetes Typ 2 - vom Zielkorridor zum Patient

Need“, Köln, 17. Oktober 2013.

Dieser Text entstand mit freundlicher

Unterstützung durch Novo Nordisk Pharma

$\mathrm{GmbH}$, Mainz

Tresiba $^{\circledast} 100$ Einheiten/ml Injektionslösung in einem Fertigpen (FlexTouch ${ }^{\circledast}$ ). Tresiba ${ }^{\circledR} 200$ Einheiten/ml Injektionslösung in einem Fertigpen (FlexTouch ${ }^{\circledR}$ ). Tresiba ${ }^{\circledR} 100$ Einheiten $\mathrm{ml}$ Injektionslösung in einer Patrone (Penfill ${ }^{\otimes}$. Wirkstoff: Insulin degludec. Zusammensetzung: Arzneilich wirksamer Bestandteil: 100/200 E/ml Insulin degludec, gentechnisch hergestellt in Saccharomyces cerevisiae mit Hilfe von rekombinanter DNS. Sonstige Bestandteile: Glycerol, Metacresol, Phenol, Zinkacetat, Salzsäure (zur Einstellung des pH-Werts), Natriumhydroxid (zur Einstellung des pH-Werts), Wasser für Injektionszwecke. Anwendungsgebiete: Diabetes mellitus bei Erwachsenen. Auch in Kombination mit oralen Antidiabetika und schnell wirkenden Insulinen. Art der Anwendung: Nur zur s.c. Injektion. Tresiba ${ }^{\circledR}$ steht in 2 Stärken zur Verfügung, die Dosisanzeige zeigt stets die Anzahl der Einheiten, daher darf keine Dosisumrechnung bei Umstellung der Stärke vorgenommen werden. Bei Tresiba ${ }^{\circledR} 200$ Einheiten/ml darf nicht mit einer Insulinspritze Insulin aus dem Fertigpen entnommen werden. Gegenanzeigen: Überempfindlichkeit gegen Insulin degludec oder einen der sonstigen Bestandteile. Vorsicht bei der Kombination mit Pioglitazon (Spontanmeldungen von Herzinsuffizienz). Es liegen keine Daten über die Anwendung von Tresiba ${ }^{\circledR}$ in der Schwangerschaft oder Stillzeit sowie bei Kindern und Jugendlichen unter 18 Jahren vor. Es ist nicht bekannt, ob Insulin degludec in die Muttermilch übergeht. Nebenwirkungen: Hypoglykämien. Ödeme zu Beginn der Behandlung. Reaktionen an der Injektionsstelle (Rötung, Schwellung, Entzündungen, Juckreiz und Blutergüsse). Lipodystrophien an der Injektionsstelle. Allergische Reaktionen, potenziell allergische Reaktionen, Urtikaria und Ausschläge; sehr selten generalisierte Überempfindlichkeitsreaktionen, die lebensbedrohlich sein können. Bei schneller Verbesserung der Blutzuckereinstellung vorübergehende Verschlechterung der diabet. Retinopathie. Verschreibungspflichtig. Novo Nordisk A/S, Novo Allé, 2880 Bagsvaerd, Dänemark. Stand: Mai 2013

Victoza $^{\circledR} 6 \mathrm{mg} / \mathrm{ml}$ Injektionslösung in einem Fertigpen. Wirkstoff: Liraglutid. Zusammensetzung: Arzneilich wirksamer Bestandteil: $6 \mathrm{mg} / \mathrm{ml}$ Liraglutid. Analogon zu humanem Glucagonlike peptide-1 (GLP-1), hergestellt durch rekombinante DNS-Technologie in Saccharomyces cerevisiae. Sonstige Bestandteile: Natriummonohydrogenphosphat-Dihydrat, Propylenglykol, Phenol, Wasser für Injektionszwecke. Anwendungsgebiete: Behandlung des Diabetes mellitus Typ 2 bei Erwachsenen in Kombination mit Metformin und/oder einem Sulfonylharnstoff oder in Kombination mit Metformin und einem Thiazolidindion, wenn trotz maximal verträglicher Dosis bei der Metformin- oder Sulfonylharnstoff-Monotherapie oder trotz Therapie mit 2 oralen Antidiabetika keine ausreichende Blutzuckerkontrolle erreicht werden kann. Art der Anwendung: Der Pen wurde für die Verwendung mit NovoFine ${ }^{\circledR}$ oder NovoTwist ${ }^{\circledR}$ EinwegInjektionsnadeln entwickelt. Gegenanzeigen: Überempfindlichkeit gegen den Wirkstoff oder einen der sonstigen Bestandteile, bestehende oder geplante Schwangerschaft, Stillzeit, Typ 1 Diabetes, diabetische Ketoazidose, Patienten unter 18 lahren Die Anwendung von Victoz ${ }^{\circledR}$ wird bei mittelschwerer und schwerer Nierenfunktionsstörung einschließlich terminaler Niereninsuffizienz, Leberfunktionsstörung, entzündlichen Darmkrankheiten und diabetischer Gastroparese Patienten mit bereits bestehender Insulintherapie nicht empfohlen. Bei Niereninsuffizienz, Leberfunktionsstörung, entzündlichen Darmkrankheiten und diabetischer Gastroparese Patienten mit bereits bestehender Insulintherapie nicht empfohlen. Bei
Verdacht auf eine Pankreatitis ist Victoza ${ }^{\circledR}$ abzusetzen. Nebenwirkungen: Sehr häufig: Kopfschmerzen, vorübergehende Übelkeit u. Durchfall (mit Gefahr der Dehydrierung). Häufig: Hypoglykämie, Erbrechen, Verstopfung, abdominale Beschwerden (z. B. Schmerzen und Spannungsgefühl), Dyspepsie, Nasopharyngitis, Bronchitis, Anorexie, verminderter Appetit, Schwindel, Gastritis, virale Gastroenteritis, Blähungen, gastroösophageale Refluxkrankheit, Zahnschmerzen, Erschöpfung, Fieber, Antikörperbildung, Reaktionen an der Injektionsstelle, Ausschlag. Gelegentlich: Neoplasmen der Schilddrüse, Erhöhung der Calcitonin-Konzentration im Blut, Struma, Urtikaria, Unwohlsein, Dehydrierung mit Verschlechterung der Nierenfunktion, akutes Nierenversagen, Juckreiz. Selten: Angioödem, anaphylakt. Reaktion. Sehr selten: Pankreatitis (einschl. nekrotisierende Pankreatitis). Häufigkeit nicht abschätzbar: Erhöhter Puls. Verschreibungspflichtig. Novo Nordisk A/S, Novo Allé, 2880 Bagsvaerd, Dänemark. Stand: März 2013

Levemir ${ }^{\circledR} 100 \mathrm{E} / \mathrm{ml}$ Injektionslösung in einem Fertigpen (FlexPen ${ }^{\circledR}$ ). Levemir ${ }^{\circledR} 100 \mathrm{E} / \mathrm{ml}$ Injektionslösung in einer Patrone (Penfill ${ }^{\circledR}$ ). Wirkstoff: Insulin detemir. Zusammensetzung: Arzneilich wirksamer Bestandteil: $100 \mathrm{E} / \mathrm{ml}$ Insulin detemir, gentechnisch hergestellt aus rekombinanter DNS in Saccharomyces cerevisiae. Sonstige Bestandteile: Glycerol, Phenol, Metacresol, Zinkacetat, Natriummonohydrogenphosphat-Dihydrat, Natriumchlorid, Salzsäure, Natriumhydroxid, Wasser für Injektionszwecke. Anwendungsgebiete: Diabetes mellitus bei Erwachsenen, Jugendlichen und Kindern ab 2 Jahren. Auch in Kombination mit oralen Antidiabetika, schnell wirkenden Insulinen oder als Zusatzmedikation zu Liraglutid (Victoza ${ }^{\circledR}$ ). Art der Anwendung: Nur zur s. c. Injektion. Gegenanzeigen: Überempfindlichkeit gegen Insulin detemir oder einen der sonstigen Bestandteile. Vorsicht bei der Kombination mit Pioglitazon (Spontanmeldungen von Herzinsuffizienz). Eine Behandlung während der Schwangerschaft kann unter Abwägung des potentiellen Nutzens gegen das möglicherweise erhöhte Risiko eines ungünstigen Schwangerschaftsausgangs in Betracht gezogen werden. Es ist nicht bekannt, ob Insulin detemir in die Muttermilch übergeht. Ggf. ist in Schwangerschaft und Stillzeit eine Dosisanpassung notwendig. Nebenwirkungen: Hypoglykämien. Sehstörungen oder Ödeme zu Beginn der Behandlung. Reaktionen an der Injektionsstelle (Rötung, Schwellung, Entzündungen, Juckreiz und Blutergüsse). Lipodystrophien an der Injektionsstelle. Allergische Reaktionen, potenziell allergische Reaktionen, Urtikaria und Ausschläge; sehr selten generalisierte Überempfindlichkeitsreaktionen, die lebensbedrohlich sein können. Bei schneller Verbesserung der Blutzuckereinstellung vorübergehende Verschlechterung der diabet. Retinopathie und revers. akute schmerzhafte Neuropathie. Verschreibungspflichtig. Novo Nordisk A/S, Novo Allé, 2880 Bagsvaerd, Dänemark. Stand: März 2012 


\section{Innovative Substanzgruppe für Typ-2-Diabetiker}

Dass auch die Niere an der Regulation des Blutzuckers beteiligt ist, wurde lange Zeit eher beiläufig zur Kenntnis genommen. Es ist jedoch gesichert, dass speziell beim Typ-2-Diabetiker der im proximalen Tubulus lokalisierte Sodium GLucose Transporter 2 (SGLT 2) eine deutliche renale Reabsorption der Glukose bewirkt und damit zur Hyperglykämie beiträgt. Aus der Blockade dieses Transporters hat sich ein neuer und vielversprechender therapeutischer Ansatz entwickelt. Zu den klinischen Effekten der SGLT-2-Inhibition, die eine kontrollierte Glukosurie erzeugt, zählen die deutliche Senkung des $\mathrm{HbA}_{1 c^{-}}{ }^{-}$ Spiegels, die spürbare Gewichtsabnahme und die dank der geringeren Natriumbelastung bedingte Reduktion des systolischen wie diastolischen Blutdrucks, berichtete Prof. John Wilding, Liverpool.
SGLT-2-Inhibitoren wie Canagliflozin (Invokana ${ }^{\circledR}$ ) können als Monotherapeutika, aber auch als Kombinationspartner eingesetzt werden. Behandelt man einen Typ-2-Diabetiker von Anfang an mit einer Kombination, die einen SGLT-2-Hemmer enthält, ist eine Senkung des $\mathrm{HbA}_{1 \mathrm{c}}$ um bis zu 2,0 Prozentpunkte möglich, konstatierte mit Nachdruck Prof. Julio Rosenstock, Dallas, Texas. Seiner Einschätzung nach eignen sich die innovativen Pharmaka zur individualisierten Therapie aller Typ-2Diabetiker. Sie brauchen nur einmal täglich appliziert zu werden, entfalten ihren antidiabetischen Effekt unabhängig von einer Insulinresistenz und haben ein sehr geringes Hypoglykämierisiko.

Sind Typ-2-Diabetiker trotz maximal effektiver Dosen von Metformin und einem Sulfonylharnstoff unzureichend einge- stellt, profitieren sie von der zusätzlichen Gabe von Canagliflozin. Das ergab eine randomisierte, doppelblinde und placebokontrollierte Phase-III-Studie mit 469 Erwachsenen und einer Laufzeit von 52 Wochen. Unter einmal täglich $100 \mathrm{mg}$ Canagliflozin fiel der $\mathrm{HbA}_{1 c}$-Wert um 0,74 und unter $300 \mathrm{mg}$ um 0,96 Prozentpunkte ab. In der Placebogruppe betrug die Veränderung lediglich 0,01 Prozentpunkte. Beobachtet wurden ferner eine Abnahme des Körpergewichts um 2,2 bzw. 3,2\% sowie des systolischen Blutdrucks um 3,7 bzw. 2,9 mmHg.

Karl B. Filip, Landsberg

Quelle: Symposium „New options and opportunities in the era of individualized therapy“ im Rahmen der 49. Jahrestagung der EASD am 23. September 2013 in Barcelona. Veranstalter: Janssen Pharmaceutica NV

\section{Jeder achte Mensch mit Diabetes leidet auch an einer Depression}

Das Leben mit Diabetes ist für viele Menschen eine ernstzunehmende Belastung, gerade weil sie stets gezwungen sind, die Therapie ihrem privaten und beruflichen Tagesablauf anzupassen. Auf Belastungen im Umgang mit der Erkrankung, wie beispielsweise schwankende Blutzuckerwerte, Folgekomplikationen oder Hypoglykämien, reagieren Menschen mit Diabetes oftmals mit depressiven Symptomen wie Niedergeschlagenheit, Antriebslosigkeit, Angstgefühlen, Schlafstörungen oder körperlichen Schmerzen. So weisen Patienten beispielsweise in den ersten 30 Tagen nach einer schweren Hypoglykämie eine erhöhte depressive Symptomatik auf. US-Wissenschaftler ermittelten ein sehr stark erhöhtes Risiko für Menschen mit Diabetes, an einer Depression zu erkranken, und konnten dabei eine Korrelation zur Art der Therapie aufzeigen: In einer Studie, die mehr als 65000 Frauen mit Diabetes über einen Zeitraum von 10 Jahren untersuchte, wurde bei Probandinnen, die mit oralen Antidiabetika behandelt wurden, ein um $25 \%$ erhöhtes Risiko festgestellt - insulinbehandelte Patientinnen waren sogar um $53 \%$ stärker gefährdet, eine Depression zu entwickeln.
Teufelskreis: Wechselwirkung zwischen Diabetes und Depression

Umgekehrt erhöht die Depression das Risiko für einen ungünstigen Verlauf des Diabetes: Sie erschwert das Management des Diabetes hinsichtlich regelmäßiger Blutzuckerkontrollen, reduziert die Adhärenz und verhindert das Erreichen wichtiger Therapieziele. Untersuchungen belegen eine erhöhte Gefährdung für die Entwicklung von Folgekomplikationen sowie ein erhöhtes Mortalitätsrisiko, wenn Diabetes und Depression gemeinsam auftreten.

Depression frühzeitig diagnostizieren Um bei Menschen mit Diabetes schlechte Blutzuckerwerte und Folgekomplikationen zu vermeiden, ist es elementar, eine erhöhte Depressivität oder eine klinische Depression frühzeitig zu erkennen und zu behandeln. Experten schätzen, dass nur die Hälfte der depressiven Menschen mit Diabetes überhaupt die richtige Diagnose erhält. Das zentrale diagnostische Instrument ist das Arzt-Patienten-Gespräch. Rechtzeitig diagnostiziert, sind Depressionen gut therapierbar: Die im Sommer 2013 aktualisierte S2-Leitlinie „Psychosoziales und Diabetes“ empfiehlt bei leichter Symptomatik eine psychosomatische Basisversorgung durch den Hausarzt, Internisten oder Diabetologen, bei mittelschwerer und schwerer Depression soll die Kombination einer Pharmakotherapie mit einer Psychotherapie geprüft werden. Bei einer schweren Depression ist zusätzlich die Integration eines Facharztes in die Therapie wichtig.

Einfache und sichere Blutzuckerselbstkontrolle im Alltag

Die regelmäßige und exakte Kontrolle des Blutzuckers ist für Menschen mit Diabetes wichtig. Je besser sich die Blutzuckerselbstkontrolle in den Alltag integrieren lässt und je einfacher das Blutzuckermessgerät $\mathrm{zu}$ bedienen ist, desto weniger belastet das Diabetesmanagement die Betroffenen. Die Bestimmung des Blutzuckerwerts durch einfache und zugleich hochpräzise Messgeräte wie die Contour Next Systeme ${ }^{\circledR}$ (Contour Next, Contour XT oder Contour Next USB) kann dies leisten. Eine gute Blutzuckereinstellung mit geringen Schwankungen der Werte kann dazu beitragen, die Lebensqualität von Menschen mit Diabetes zu steigern.

Pressemitteilung Bayer HealthCare 


\section{Therapie mit DPP-4-Hemmer ist sicher}

Bisher herrschte Unklarheit darüber, ob alle Therapien zur Kontrolle des hohen Blutzuckers kardiovaskulär sicher sind, oder ob sie sogar geeignet sind, Komplikationen wie Myokardinfarkt oder Schlaganfall zu reduzieren, erklärte Prof. Deepak L. Bhatt, Boston. SAVOR-TIMI-53 sollte hierzu neue Erkenntnisse bringen. Die Phase-IV-Studie war auf Nichtunterlegenheit des DPP-4 (Dipeptidyl Peptidase 4)-Hemmers gegenüber Placebo bezüglich des kombinierten Endpunkts aus kardiovaskulärem Tod, nicht-tödlichem Herzinfarkt und nicht-tödlichem Schlaganfall angelegt. Es handelte sich dabei um eine Behandlung zusätzlich zu einer antihyperglykämischen Standardtherapie.

Die insgesamt 16492 beteiligten Patienten besaßen ein hohes kardiovaskuläres Risiko, entweder wegen einer kardiovaskulären Erkrankung oder bedingt durch multiple Risikofaktoren wie hohes Alter, Dyslipidämie, Hochdruck oder Rauchen. Saxagliptin (Onglyza ${ }^{\circledR}$ ) wurde mit $5 \mathrm{mg}$ täglich oder bei Niereninsuffizienz in halbierter Dosis verabreicht.

Nichtunterlegenheit belegt

In der Saxagliptin-Gruppe verringerte $\circ$ Hypoglyämien traten unter Saxagliptin sich der $\mathrm{HbA}_{1 \mathrm{c}}$-Wert über die Zeit signifi- 둘 signifikant häufiger auf (15,3 vs. 13,4\%, kant stärker, dementsprechend erreich- $\mathrm{p}<0,001$ ), verursachten aber nicht mehr ten auch signifikant mehr Patienten den $\stackrel{\circ}{\frac{.0}{0}}$ Aufenthalte im Krankenhaus. Schwere Zielwert $<7 \%$ (40,0 vs. 30,0\% nach 2 Jahren).

Der primäre kardiovaskuläre Endpunkt war nach 2 Jahren bei 7,3\% (Saxagliptin) versus $7,2 \%$ (Placebo) der Patienten eingetreten $(p<0,001)$. Der Nachweis der Nichtunterlegenheit war damit erbracht. Der sekundäre Endpunkt, bestehend aus dem primären Endpunkt plus Hospitalisierung aufgrund von Herzinsuffizienz, instabiler Angina sowie koronarer Revaskularisierung differierte mit $12,8 \%$ (Saxagliptin) beziehungsweise $12,4 \%$ (Placebo) ebenfalls nicht signifikant. Lediglich Fälle von Hospitalisierung wegen Herzinsuffizienz waren unter dem DPP-4-Hemmer häufiger (3,5 vs. $2,8 \%, p=0,007$ ). $\ddot{t}$ veränderungen, Frakturen oder Tumoren unterschieden sich zwischen Saxagliptin und Placebo nicht signifikant. Spezielles Interesse galt dem Auftreten von Pankreatitiden. Saxagliptin erhöhte das Risiko sowohl einer Pankreatitis oder eines Pankreaskarzinoms nicht. Vielmehr kam es unter Placebo zu 12, unter Saxagliptin nur zu 5 derartigen Karzinomen.

Martin Bischoff, Planegg

Quelle: Hot Line Session „SAVOR-TIMI 53:

Saxagliptin Assessment of Vascular Outcomes Recorded in Patients with Diabetes mellitus", am 2. September 2013 anlässlich des ESC-Kongresses in Amsterdam. Veranstalter: Bristol-Myers Squibb $\mathrm{GmbH} \&$ Co.KgaA, München, und Astra Zeneca $\mathrm{GmbH}$, Wedel.

\section{Schulung für türkischstämmige Typ-2-Diabetiker}

Laut Schätzungen leben etwa 600000 Migranten mit Diabetes in Deutschland [1]. Die meisten davon sind türkischer Herkunft. Viele von ihnen haben gegenüber der deutschstämmigen Bevölkerung aufgrund von fehlenden Informationen sowie sprachlichen und kulturellen Barrieren einen schlechteren Zugang zum Gesundheitssystem. Deshalb bietet seit 4 Jahren das Pharmaunternehmen Lilly in Kooperation mit Partnern die Schulungsinitiative „Diabetes gemeinsam verstehen“ an. Nun können durch eine Kooperation mit der Diabetologen Hessen eG sowie der Türkisch-Deutschen Gesundheitsstiftung e.V. auch Patienten in Hessen von den Diabetesschulungen profitieren.

\section{Migranten profitieren von spezieller Schulung}

Die Kooperation ist bislang einzigartig. „Als Genossenschaft der Diabetologen in Hessen liegt uns eine gute Versorgung unserer Patienten besonders am Herzen. Migranten sind da häufig benachteiligt.
Daher ist diese Kooperation ein großer Gewinn“, sagte PD Dr. Klaus Ehlenz, Vorstandsvorsitzender der Diabetologen Hessen eG.

Wenn den Patienten dabei geholfen wird, ein besseres Leben mit Diabetes zu führen, haben wir schon viel gewonnen“, betonte Dr. Yasar Bilgin, erster Vorsitzender der Türkisch-Deutschen Gesundheitsstiftung e.V. „Die bildhafte, interaktive Wissensvermittlung anhand von Gesprächslandkarten macht die Schulung lebendig und hilft dabei, soziale und kulturelle Barrieren zu überwinden.“

\section{Herausforderung Ramadan}

Die wohl größte Herausforderung stellt für die meisten Patienten der Ramadan dar. Muslime mit Diabetes sind als chronisch Kranke nicht zum Fasten verpflichtet; dennoch möchten viele den Ramadan einhalten. Eine neue Gesprächslandkarte zum Umgang mit dem Diabetes während des Ramadan wird daher künftiger Bestandteil der Schulungen sein.
Ergebnisse einer Befragung bei den hessischen Patienten zeigten, dass sich bei fast allen der Wissensstand verbessert hat [2]. Damit konnten die bereits vorliegenden Ergebnisse einer Untersuchung bestätigt werden, in der die türkischstämmigen Patienten ein der deutschen Vergleichsgruppe entsprechendes Wissensniveau erreichten [3]. „Erstmals verstehen sie, was Diabetes wirklich ist und verändern ihre Gewohnheiten langfristig“, berichtete Ayse Ünal, Diabetesberaterin aus Gelsenkirchen.

Dr. Ralph Hausmann, Frankfurt

Literatur

1 Icks $A$ et al. Deutscher Gesundheitsbericht Diabetes. DDU (Hrsg). 2011; 148-154

2 Diabetologen Hessen eG. Evaluation „Diabetes gemeinsam verstehen in Hessen", 2013

3 Korolewa $\mathrm{V}$ et al. DDG. Berlin, 4.-6. November 2010; 115-Poster

Quelle: Forum Diabetes und Migration: „Diabetes gemeinsam verstehen“ in Hessen am 9. Oktober 2013 in Bad Homburg. Veranstalter: Lilly Deutschland 


\section{Neue Optionen bereichern antidiabetische Therapie}

Nach einhelliger Einschätzung der Experten bedeuten die vor wenigen Jahren eingeführten GLP-1-Rezeptoragonisten eine deutliche Bereicherung der therapeutischen Optionen. Diese per injectionem zu applizierenden Inkretinmimetika zeichnen sich dadurch aus, dass sie die Freisetzung von Insulin in strenger Abhängigkeit von der jeweils aktuellen Konzentration des Blutzuckers regulieren. GLP-1-Rezeptoragonisten sind als Monotherapeutika einsetzbar, können aber auch mit oralen Antidiabetika sowie mit Insulin kombiniert werden. Einer der neuesten Vertreter dieser innovativen Substanzklasse ist das in den USA bereits zugelassene Dulaglutid. Dessen Wirksamkeit und Sicherheit werden in einem umfangreichen Studienprogramm „Assessment of Weekly AdministRation of LY2189265 in Diabetes“ (AWARD) überprüft und jeweils mit einer etablierten Substanz zur Therapie des Typ-2-Diabetes verglichen, war auf der 49. Jahrestagung der European Association for the Study of Diabetes (EASD) Ende September zu erfahren. In der AWARD-1-Studie, der ersten von insgesamt 8, verglich man an 978 Typ-2-Diabetikern, die mit Metformin und Pioglitazon therapiert wurden, die Wirksamkeit der zusätzlichen Gabe von Dulaglutid, das einmal wöchentlich zu verabreichen ist, mit Exenatid und Placebo.

Die Ergebnisse sprechen für sich: Bereits nach 26 Wochen zeigte sich bei der Senkung des $\mathrm{HbA}_{1 \mathrm{c}}$-Werts die Überlegenheit des GLP-1-Rezeptoragonisten gegenüber Exenatid und Placebo. Die Reduktion betrug 1,51 Prozentpunkte unter $1,5 \mathrm{mg}$ und 1,30 unter $0,75 \mathrm{mg}$ Dulaglutid sowie 0,99 und 0,46 Prozentpunkte unter Exenatid bzw. Placebo. Als durchaus will- kommener Nebeneffekt fand sich unter 1,5 mg Dulaglutid eine Gewichtsabnahme um 1,4 kg und unter Exenatid um $1,14 \mathrm{~kg}$. Die häufigsten Nebenwirkungen beider Pharmaka waren gastrointestinaler Art. Schwere Hypoglykämien wurden nicht beobachtet. In der AWARD-3-Studie mit 807 Typ-2-Diabetikern, die einen $\mathrm{HbA}_{1 \mathrm{c}}$-Ausgangswert von 7,6\% aufwiesen, bewirkten 1,5 mg Dulaglutid einmal wöchentlich einen Rückgang um 0,78 Prozentpunkte. Unter der Vergleichssubstanz Metformin fiel der Wert nur um 0,56 Prozentpunkte. Bei beiden Pharmaka nahmen die Patienten innerhalb von 26 Wochen jeweils mehr als $2 \mathrm{~kg}$ ab.

Karl B. Filip, Landsberg

Quelle: Media Briefing „The Art of GLP-1Therapy", im Rahmen der 49. Jahrestagung der EASD am 24. September 2013 in Barcelona. Veranstalter: Lilly Diabetes

\section{Neuer Service erleichtert Einstieg in die Insulinpumpentherapie}

Die Genehmigung einer Insulinpumpe unterliegt strengen Indikationskriterien wie beispielsweise häufigen Hypoglykämien oder einem ausgeprägten DawnPhänomen. Für eine optimale Prüfung durch die Krankenkasse müssen Patienten für das CSII-Genehmigungsverfahren ein lückenloses, über 3-4 Monate geführtes Tagebuch mit sämtlichen Blutzuckerwerten und Insulingaben einreichen. Um das Verfahren erfolgreich zu durchlaufen, ist es außerdem notwendig, die Diabetesdaten und das individuelle Gutachten des Arztes nach speziellen Vorgaben der Krankenkassen zusammenzustellen. Entsprechend hoch ist der Zeit- und Arbeitsaufwand für Praxen und Patienten. Aufgrund der umfangreichen Datensammlung ist dieser Prozess außerdem oft fehleranfällig.

Aktionspaket unterstützt Ärzte und Patienten

Um Ärzte und Patienten während der Genehmigungsphase umfassend zu unterstützen, wurde die Aktion „Start mit AccuChek $^{\circledR “}$ initiiert. Hierfür erhalten Diabetologen eine Gutachten-Software sowie das Aktionspaket mit umfangreichen Unter- lagen und dem Accu-Chek ${ }^{\circledR}$ Aviva Expert Blutzuckermessgerät mit integriertem Bolusrechner zur Abgabe an den Patienten. Nach gemeinsamer Besprechung der Unterlagen erfolgt die Schulung des Patienten auf den Bolusrechner und das dazugehörige Datenmanagement. Ein aufwendiges handschriftliches Tagebuch mit seinen potenziellen Fehlerquellen wird nicht mehr benötigt. Stattdessen kann die Dokumentation komplett elektronisch erfolgen, denn das Blutzuckermessgerät speichert sowohl die Blutzuckermesswerte als auch die Insulingaben, Kohlenhydratwerte und Ereignisse wie z.B. Sport oder Stress. Der Patient wird lediglich gebeten, ergänzend ein Dokumentationsheft zur Erfassung außergewöhnlicher Ereignisse - beispielsweise Krankheiten - zu nutzen. Eine Checkliste gibt zudem Übersicht über alle notwendigen Schritte für die erfolgreiche Beantragung einer Insulinpumpe. Die spätere Einreichung wird somit optimal vorbereitet.

Elektronische Gutachtenerstellung

Herzstück für den Arzt ist die neue CSII Gutachten Software, die alle erforderlichen Bestandteile zur Gutachtenerstel- lung beinhaltet. Das elektronische Tagebuch der Patienten mit der vollständigen Dokumentation aus dem Blutzuckermessgerät wird via Accu-Chek ${ }^{\circledR}$ Smart Pix Software in die Gutachtensoftware exportiert. Per Knopfdruck werden die Angaben der Patienten auf Vollständigkeit und Plausibilität geprüft: So wird bei männlichen Patienten beispielsweise Gestationsdiabetes automatisch ausgeschlossen. Im nächsten Schritt erstellt der Arzt mithilfe von Textbausteinen aus der Software das Gutachten auf Basis der kumulierten Daten. Der anschließende Export des Gutachtens in das Krankenkassenformular erfolgt automatisch, das manuelle Ausfüllen von Formularen entfällt. Die aktuellen Richtlinien (Stand: August 2012) des Medizinischen Dienstes der Krankenkassen (MDK) werden dabei voll berücksichtigt. Auf diese Weise erfolgt eine deutliche Zeitersparnis und die Gutachtenerstellung für den Arzt wird vereinfacht. Bei Patienten mit entsprechender Indikation kann durch die vollständige Datenbasis die Wahrscheinlichkeit von Widersprüchen durch den MDK reduziert werden.

Pressemitteilung Roche Diagnostics Deutschland $\mathrm{GmbH}$ 